\title{
Dysbiosis of urine microbiota in obstructive urinary retention patients revealed by next-generation sequencing
}

Shan Jiang ${ }^{1 \dagger}$, Saisai Lu ${ }^{2 \dagger}$, Xiaomin Chen ${ }^{1}$, Fengxia Li ${ }^{1}$, Chengwei Zhu ${ }^{2}$, Yuancai Zheng ${ }^{3}$, Xiaobing Wang ${ }^{2 *}$ and Shihao $\mathrm{Xu}^{4^{*}}$

\begin{abstract}
Background: Urinary retention (UR) is a common urinary system disease can be caused by urinary tract obstruction with numerous reasons, however, the role of urine microbes in these disorders is still poorly understood. The aim of this study was to identify the urine microbial features of two common types of obstructive UR, caused by urinary stones or urinary tract tumors, with comparison to healthy controls.

Methods: Urine samples were collected from a cohort of 32 individuals with stone UR, 25 subjects with tumor UR and 25 healthy controls. The urine microbiome of all samples was analyzed using high-throughput $16 \mathrm{~S}$ rRNA (16S ribosomal RNA) gene sequencing.

Results: We observed dramatically increased urine microbial richness and diversity in both obstructive UR groups compared to healthy controls. Despite different origins of UR, bacteria such as Pseudomonas, Acinetobacter and Sphingomonas were enriched, while Lactobacillus, Streptococcus, Gardnerella, Prevotella and Atopobium were decreased in both UR groups in comparison with healthy controls, exhibited an approximate urine microbial community and functional characteristics of two types of obstructive UR. Furthermore, disease classifiers were constructed using specific enriched genera in UR, which can distinguish stone UR or tumor UR patients from healthy controls with an accuracy of $92.29 \%$ and $97.96 \%$, respectively.

Conclusion: We presented comprehensive microbial landscapes of two common types of obstructive urinary retention and demonstrated that urine microbial features of these patients are significantly different from that of healthy people. The urine microbial signatures would shed light on the pathogenesis of these types of urinary retention and might be used as potential classification tools in the future.
\end{abstract}

Keywords: Urine microbiome, Obstructive urinary retention, $16 \mathrm{~S}$ rRNA gene sequencing

*Correspondence: gale820907@163.com; dcxshvip@wmu.edu.cn

tShan Jiang and Saisai Lu contributed equally to this work. Author order was determined by alphabetically

2 Department of Rheumatology, The First Affiliated Hospital of Wenzhou Medical University, Wenzhou, China

${ }^{4}$ Department of Ultrasonography, The First Affiliated Hospital of Wenzhou Medical University, Wenzhou, China

Full list of author information is available at the end of the article

\section{Background}

Urinary retention (UR) is a series of complex diseases defined as the inability to completely empty the bladder due to various causes [1-3]. UR affects both men and women; however, it presents with a male to female ratio of 10:1 [4] due to the high presence of prostate gland problems in males [5]. UR can be classified into nonobstructive and obstructive types according to the cause of its formation. Nonobstructive causes include medications, bladder muscle weakness and nerve problems that

c) The Author(s) 2021. This article is licensed under a Creative Commons Attribution 4.0 International License, which permits use, sharing, adaptation, distribution and reproduction in any medium or format, as long as you give appropriate credit to the original author(s) and the source, provide a link to the Creative Commons licence, and indicate if changes were made. The images or other third party material in this article are included in the article's Creative Commons licence, unless indicated otherwise in a credit line to the material. If material is not included in the article's Creative Commons licence and your intended use is not permitted by statutory regulation or exceeds the permitted use, you will need to obtain permission directly from the copyright holder. To view a copy of this licence, visit http://creativeco mmons.org/licenses/by/4.0/. The Creative Commons Public Domain Dedication waiver (http://creativecommons.org/publicdomain/ zero/1.0/) applies to the data made available in this article, unless otherwise stated in a credit line to the data. 
interfere with the brain's ability to receive signals that the bladder is full and the body's ability to function properly [6-8]. Conditions such as benign prostatic hyperplasia (BPH), urinary tract stones and certain tumors can lead to urethral stricture or deformation cause obstruction [9]. UR can also be chronic or acute. Chronic UR can be a long-lasting and less painful medical condition, still resulting in urinary incontinence (UI), a urinary tract infection (UTI), and so on. In contrast, acute UR occurs suddenly and causes great discomfort or pain to the patient. It is a potentially life-threatening medical condition that requires immediate emergency treatment, such as bladder drainage, urethral dilation or surgery.

Increasing studies have revealed that bacteria not only exist in the urine tracts of healthy individuals but play a crucial role in the maintenance of microecological balance [10-13]. In addition, with the advent of cultureindependent methods for detect microbe, amount of studies proved that the abnormal urine microbial community is also closely related with diverse urologic disorders. UTI accounts for a high proportion of these population, with the most common microbe being Escherichia coli followed by some gram-positive cocci and other Enterobacteriaceae [14]. In 2014, Meghan et al. used the expanded quantitative urine culture (EQUC) techniques observed that nine bacteria (Actinobaculum, Actinomyces, Aerococcus, Arthrobacter, Corynebacterium, Gardnerella, Oligella, Staphylococcus, and Streptococcus) were obviously exited in the urine of Urgency urinary incontinence (UUI) patients [15]. This team also revealed that the UUI urine microbiome consisted of increased Gardnerella and decreased Lactobacillus compared to the non-UUI group based on 16S rRNA gene sequencing [15]. Recent work by Siddiqui et al. found that more than $90 \%$ of sequencing reads in the urine of interstitial cystitis (IC) patients were belong to Lactobacillus, while $60 \%$ in healthy female urine [16]. Sequencing of bacteria-specific $16 \mathrm{~S}$ rDNA in the mid-stream urine of 25 chronic prostatitis/chronic pelvic pain syndrome (CP/CPPS) patients and 25 asymptomatic or only had urinary symptoms men controls, demonstrated significantly higher phylogenetic diversity in the urine microbiota of CP/CPPS patients and higher Clostridia count than the control group [17]. Dornbier et al. sequenced 16S rRNA of bladder urine and urinary stones in 52 patients, suggested that the presence of bacterial communities in non-struvite stone was relatively higher than the surrounding urine [18]. Another study recruited patients with different stone type found that there was a common imbalance between the microbiome of urinary stone disease (USD) and different pathologies [19]. In addition, a cross-sectional study investigated the urine microbiome of asymptomatic bacteriuria who had risks from a neurogenic bladder due to spinal cord injury and healthy controls, showed altered abundance of Lactobacillales, Enterobacteriales and other microorganisms and confirmed functional interactions between pathogens and human proteins in subjects who initiated host defense [20].

Based on the above information, microorganisms have been widely studied in urinary system disease, but the potential relationship between the urine microbiome and obstructive UR has not yet been fully elucidated. Therefore, we performed next-generation sequencing of $16 \mathrm{~S}$ rRNA to detect the urine microorganisms and their associated functional profile of two common obstructive UR, which were caused by stones or tumors, with comparison to that of healthy individuals. Disease classifiers were further constructed for patients with stone UR and tumor UR based on their specific microbial features.

\section{Methods}

\section{Study population}

All patients with obstructive UR and healthy controls were recruited from July 2018 to April 2019 at The First Affiliated Hospital of Wenzhou Medical University (Wenzhou, China) used for this study. Signed informed consent was obtained from each participant, for which all procedures and protocols were approved by the Medical Ethical Committee of the Wenzhou Medical University Ethics Committee. Two types of obstructive UR patients in our study were enrolled: one group presented with urinary stones, and the other group presented with obstructive UR caused by certain tumors in the urinary system that resulted in an inability to urinate automatically. The number of cases in the two groups was 34 and 25 , respectively. Patients who had taken antibiotic or probiotic treatment in the past 8 weeks were also excluded. Twenty-five healthy individuals volunteered as our control group, none of whom had a history of UTI or urinary system disease. $30 \mathrm{ml}$ fresh urine samples from all participants $(n=84)$ were collected by sterile catheter drainage under the strict aseptic procedures and treated in the same method after sampling, frozen in a sterile container at $-80^{\circ} \mathrm{C}$ within $12 \mathrm{~h}$ after sampling.

\section{Biochemical indicator detection and routine urine analysis} Blood samples were collected from all subjects to measure biochemical metabolic parameters. Approximately $3 \mathrm{ml}$ of whole blood was obtained from each volunteer's blood. Plasma samples for all analyses were obtained by centrifugation for $15 \mathrm{~min}$ at room temperature at $3000 \mathrm{rpm}$ and then frozen at $-80{ }^{\circ} \mathrm{C}$ for further analysis. Baseline biochemical indicators, including serum direct bilirubin (DBIL), indirect bilirubin (IBIL), eGFR, alkaline phosphatase (ALP) and UA, were assessed quantitatively with an automatic biochemical analyzer. Red blood cell 
count (RBC), white blood cell count (WBC), urobilinogen and proteinuria in urine samples were detected by routine urine analysis. A positive test is represented with at least one "+" sign in the column of urobilinogen or proteinuria value in the inspection report. All these clinical values were acquired based on standard procedures.

\section{DNA extraction and 16S rRNA gene amplicon sequencing}

Bacterial DNA extraction from these urine samples was performed with a TIANamp Stool DNA Kit according to the manufacturer's instructions. To distinguish the contaminants and existing microbial community, an unused clean sterile catheter was considered a negative control of sampling environment and a DNA extraction-negative control with no urine added.

The V3-V4 region of $16 \mathrm{~S}$ ribosomal RNA from the extracted DNA samples was amplified with a universal forward primer (5'-CCTACGGGNGGCWGCAG-3') and reverse primer $\left(5^{\prime}\right.$-GACTACHVGGGTATCTAATC C-3') with a single multiplex identifier and adaptors. To exclude contamination during amplification, a PCRnegative control with no template DNA were processed. PCR amplification was then parallel performed in $25 \mu \mathrm{l}$ of $2 \times$ Phanta Max Master Mix, $2 \mu \mathrm{l}$ of forward primer $(10 \mu \mathrm{M}), 2 \mu \mathrm{l}$ of reverse primer $(10 \mu \mathrm{M}), 50 \mu \mathrm{l}$ of ddH2O and template DNA. The PCR was conducted under the following conditions: $95{ }^{\circ} \mathrm{C}$ for $3 \mathrm{~min} ; 25$ cycles of $95^{\circ} \mathrm{C}$ for $30 \mathrm{~s}, 55^{\circ} \mathrm{C}$ for $30 \mathrm{~s}$, and $72{ }^{\circ} \mathrm{C}$ for $30 \mathrm{~s}$; and a final extension at $72{ }^{\circ} \mathrm{C}$ for $5 \mathrm{~min}$. Sample library and Phix sequencing control library were added, and the sequencing reaction was performed on an Illumina MiSeq sequencer and yielded $300 \mathrm{bp}$ paired-end reads at high depth.

\section{Bioinformatic analysis}

To improve the quality of analytical data, this process utilizes the quality control section from our house pipeline $[21,22]$, including to remove those sequences that did not contain primers, ambiguous reads, and reads with an average quality value $<$ Q20. Only sequences with a length longer than $300 \mathrm{bp}$ and two reads with an overlap of more than $10 \mathrm{bp}$ were merged using PANDAseq (v.2.9) [23]. The Quantitative Insights Into Microbial Ecology (QIIME) platform (v.1.9.1) [24] was used to pick the closest reference operational taxonomic unit (OTU) at a 97\% similarity cut-off, and taxonomy assignment was then mapped using the Greengenes database (v.13.8) [25]. Samples were excluded if their total reads that can be annotated to OTUs were less than 8000 , and OTUs with a number of sequences less than $0.01 \%$ of the total number of sequences were also discarded. In addition, rarefaction was performed with USEARCH [26], which randomly sampled $10-100 \%$ of the sequences from the original sequencing data to observe the number of OTUs annotated in each sequence set. Based on the OTU profile, the $\alpha$-diversity was measured using the taxa richness and diversity, and the $\beta$-diversity was estimated by computing unweighted and weighted UniFrac [27] distances and Bray-Curtis dissimilarity and further visualized with principal coordinate analysis ( $\mathrm{PCoA})$. Linear discriminant analysis (LDA) for effect size (LEfSe) program were performed based on the web services tools Galaxy (http://huttenhower.sph.harvard.edu/galaxy/root/index) [28]. The features that were significantly different among the three groups were identified by LEfSe with $\mathrm{P}<0.05$ (Kruskal-Wallis test) and LDA values $>4$ [29]. Phylogenetic Investigation of Communities by Reconstruction of Unobserved States (PICRUSt) (http://picrust.githu b.io/picrust) [30] predicted the gene family abundance from the phylogenetic information with an estimated accuracy at 0.8 , which could be used to impute the urine microbiome metagenome from the $16 \mathrm{~S}$ rRNA sequences. We selected level 3 Kyoto Encyclopedia of Genes and Genomes (KEGG) database pathways and level 2 Cluster of Orthologous Groups (COG) of abundance from the predicted functional profiles and filtered the "Function Unknown" and "Other" pathways. Only the functions and pathways with an average relative abundance $>0.01$ that existed in at least ten samples were considered in the analysis.

\section{Statistical analysis}

All statistical analyses were performed by $\mathrm{R}$ packages (version 3.4.3). The permutational multivariate analysis of variance (PERMANOVA) test was performed using the [Adonis] function of the "vegan" $\mathrm{R}$ package, with the maximum number of permutations $=999$. For comparison of all continuous variables, community diversity and richness, significantly different OTU abundance and significantly different taxon abundance at phylum and genus levels were selected using the Wilcoxon rank sum test method as previously described [31, 32]. The resulting p-values were adjusted by the Benjamini and Hochberg false discovery rate (FDR). The correlation between different genera was calculated with a Spearman correlation based on the relative abundance, which was also used to study the relationship between different bacteria and clinical indicators. For the significantly different genus abundance profile, five-fold cross-validation was performed five times on a random forest model ('randomForest' 4.6-12 package). We then obtained the average cross-validation error curve from the five processed trials. The point with the minimum crossvalidation error plus the standard deviation (SD) at the corresponding point was viewed as the cut-off point. 
We listed all sets of biomarkers with an error less than the cut-off value and chose the set with the smallest number of genera as the optimal set. To evaluate the discriminatory ability of the random forest model, we obtained the average area under the curve (AUC) and constructed the receiver operating characteristic curve (ROC) 100 times using the 'ROCR' $\mathrm{R}$ package. The mean decrease accuracy (MDA) was assigned to each feature based on the fact that removing the feature from the prediction model would increase the error rate. The probability of disease (POD) value refers to the ratio between the number of randomly generated decision tress that predicting an individual as a patient with stone UR or tumor UR and that of healthy controls. Similarly, the probability of tumor UR index is a predictive comparison between the two types of obstructive UR. The detailed script of the microbial optimal set identification and 100 times ROC analysis and POD construction can be found in the supplementary method and refer to the research published by Ren et al. in 2019 [33]. To explore which bacteria are closely related to the main enrichment functions and pathways from each group, the Spearman correlation method was also adopted.

\section{Results}

The OTU profile was obtained after 165 rDNA data analysis To study the urine microorganisms in obstructive UR, we performed $16 \mathrm{~S}$ sequencing on urine samples from 84 Chinese participants, including 34 patients with UR caused by lithiasis (stone UR), 25 patients with UR due to the urinary tract tumors (tumor UR), and 25 healthy controls. These negative controls were not sequenced, as the agarose gel electrophoresis showed that no electrophoretic band of microbial DNA, which indicates that there was no artifact and contaminant in the environment. A total of $727 \mathrm{MB}$ of $300 \mathrm{bp}$ paired-end reads were generated after sequenced on the MiSeq platform, and the average number of reads per sample was $36,072 \pm 3376$ reads (Additional file 1: Table S1). Two samples from the stone UR group, S18 and S32, were removed for their low assigned microbial reads, which were less than 8000 . Finally, we obtained 310 OTUs from 25 controls, 32 stone UR and 25 tumor UR individuals (Additional file 2: Table S2). The clinical and demographic characteristics of all remaining UR patients and controls are shown in Table 1. In terms of age, there was no significant difference between the stone UR group and control group, while the tumor UR group subjects were older than the other two groups. There was no

Table 1 Clinical characteristics of the enrolled participants

\begin{tabular}{|c|c|c|c|c|c|c|}
\hline Clinical indexes & $\begin{array}{l}\text { Control } \\
(n=25)\end{array}$ & $\begin{array}{l}\text { Stone UR } \\
(n=32)\end{array}$ & $\begin{array}{l}\text { Tumor UR } \\
(\mathrm{n}=25)\end{array}$ & $\begin{array}{l}P \text {-value Control vs } \\
\text { Stone UR }\end{array}$ & $\begin{array}{l}P \text {-value Control vs } \\
\text { Tumor UR }\end{array}$ & $\begin{array}{l}P \text {-value } \\
\text { Stone UR vs } \\
\text { Tumor UR }\end{array}$ \\
\hline Age (year) & $42.92 \pm 20.15$ & $52.94 \pm 14.40$ & $61.60 \pm 13.24$ & 0.054 & 0.0013 & 0.034 \\
\hline \multicolumn{7}{|l|}{ Gender } \\
\hline Female & $17(68 \%)$ & $21(65.62 \%)$ & $16(64 \%)$ & 1 & 1 & 1 \\
\hline Male & $8(32 \%)$ & $11(34.38 \%)$ & $9(36 \%)$ & & & \\
\hline DBIL ( $\mu \mathrm{mol} / \mathrm{L})$ & $2.50 \pm 0.70$ & $4.14 \pm 2.74$ & $4.56 \pm 5.61$ & 0.030 & 0.25 & 0.49 \\
\hline IBIL ( $\mu \mathrm{mol} / \mathrm{L})$ & $4.36 \pm 1.85$ & $6.53 \pm 2.67$ & $5.46 \pm 2.76$ & 0.043 & 0.58 & 0.12 \\
\hline eGFR & $97.56 \pm 23.70$ & $70.63 \pm 32.19$ & $40.30 \pm 33.32$ & 0.013 & 0.00010 & 0.0032 \\
\hline $\operatorname{ALP}(\mu / L)$ & $91.08 \pm 30.99$ & $91.70 \pm 33.98$ & $144.92 \pm 234.24$ & 0.96 & 0.79 & 0.81 \\
\hline $\mathrm{UA}(\mu \mathrm{mol} / \mathrm{L})$ & $317.20 \pm 54.00$ & $367.96 \pm 103.13$ & $454.01 \pm 160.95$ & 0.24 & 0.039 & 0.063 \\
\hline $\mathrm{RBC}(/ \mu \mathrm{L})$ & $9.45 \pm 10.60$ & $691.23 \pm 1348.03$ & $3489.10 \pm 6187.54$ & 0.0015 & 0.068 & 0.56 \\
\hline $\mathrm{WBC}(/ \mu \mathrm{L})$ & $55.45 \pm 123.05$ & $2110.97 \pm 5085.15$ & $1472.82 \pm 3973.84$ & 0.0096 & 0.0026 & 0.59 \\
\hline \multicolumn{7}{|l|}{ pH } \\
\hline$>6.5$ & $2(8 \%)$ & $12(37.5 \%)$ & $7(28 \%)$ & 0.27 & 0.68 & 0.56 \\
\hline$\leq 6.5$ & $9(36 \%)$ & $17(53.1 \%)$ & $16(64 \%)$ & & & \\
\hline \multicolumn{7}{|l|}{ Urobilinogen } \\
\hline Positive & $1(4 \%)$ & $3(9.4 \%)$ & $2(8 \%)$ & 1 & 1 & 1 \\
\hline Negative & $10(40 \%)$ & $27(84.4 \%)$ & $20(80 \%)$ & & & \\
\hline \multicolumn{7}{|l|}{ Proteinuria } \\
\hline Positive & $0(0 \%)$ & $20(62.5 \%)$ & 16 (64\%) & 0.00016 & 0.000086 & 0.76 \\
\hline Negative & 11 (44\%) & 10 (31.2\%) & $6(24 \%)$ & & & \\
\hline
\end{tabular}


statistically significant difference in gender among these three groups.

\section{Increased urine microbial diversity in stone UR and tumor UR individuals}

To depict the bacterial richness of each group, we randomly sampled the same amount of reads from each sample and performed rarefaction analysis to estimate the observed OTUs that could be identified from these sequences. As shown in Fig. 1a, all three curves had reached plateaus, which indicated that the amount of sequenced data were sufficient to detect the microbial feature. The acquisition rate of OTUs in control samples was strikingly lower than that in stone UR and tumor
UR groups. Measured by the Shannon index, the urine microbial diversity of the stone UR and tumor UR was significantly greater than that of healthy controls, however, there was no significant difference between the two UR groups (Fig. 1b, Shannon: controls vs stone UR, $\mathrm{P}=1.10 \times 10^{-2}$; controls vs tumor UR, $\mathrm{P}=4.57 \times 10^{-5}$; stone UR vs tumor UR, $\mathrm{P}=0.29)$. Likewise, the Simpson index of stone UR and tumor UR was significantly higher than that of controls (Fig. 1c, Simpson: controls vs stone UR, $\mathrm{P}=3.70 \times 10^{-2}$; controls vs tumor $\mathrm{UR}, \mathrm{P}=1.61 \times 10^{-3}$; stone $\mathrm{UR}$ vs tumor $\mathrm{UR}, \mathrm{P}=0.28$ ). The Chaol index and Ace index, which measure the richness of the community, showed that the richness of microbiome in two UR groups was significantly

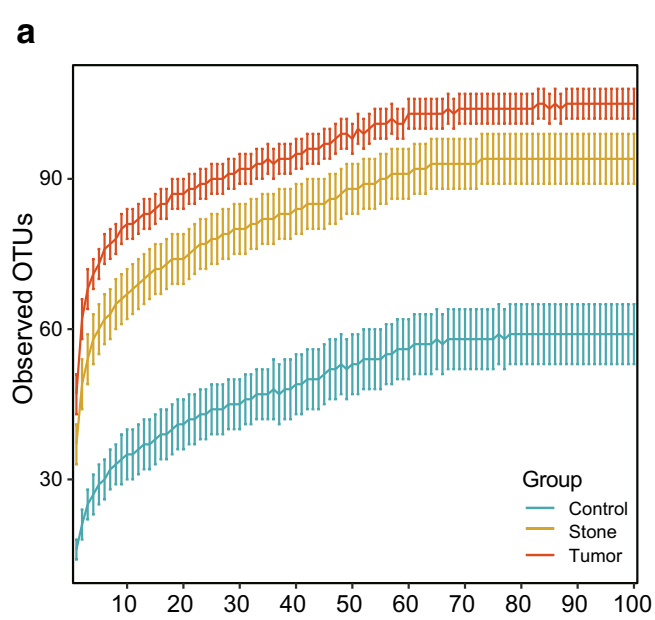

b

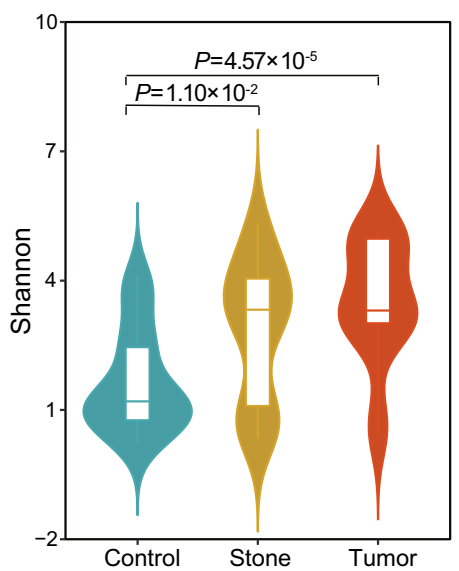

C

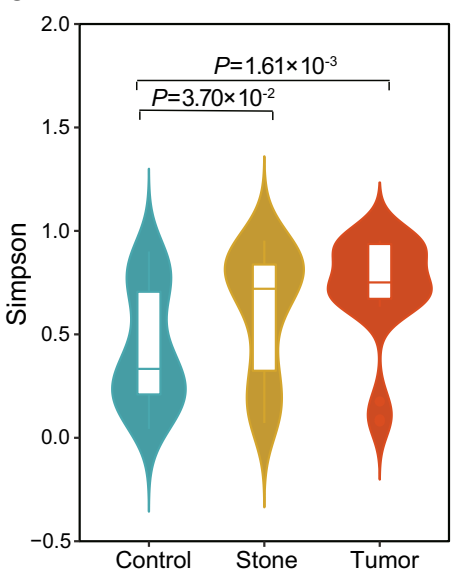

d

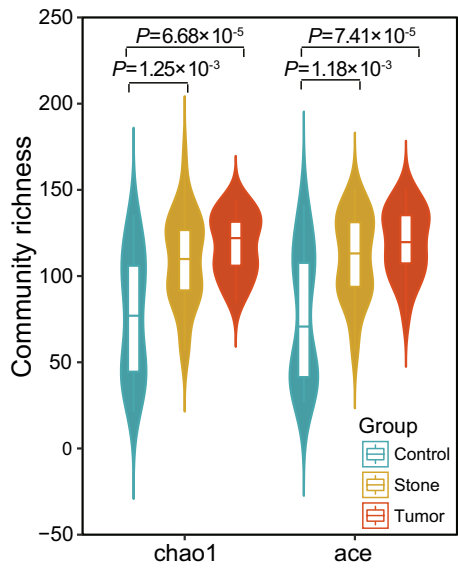

e

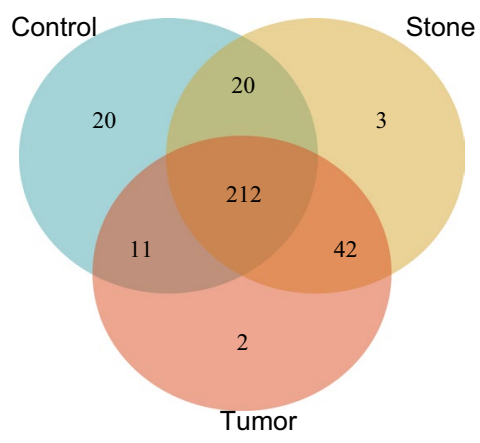

f

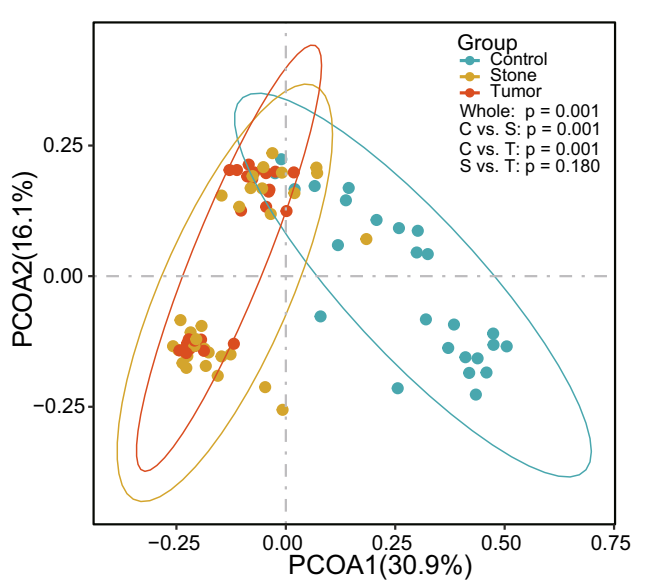

Fig. 1 Increased urine microbial diversity of stone UR and tumor UR compared with that of controls. a Rarefaction curves for observed OTU number among three groups after random sampling of 10-100\% sequences from the original sequencing data. $\mathbf{b}$ Shannon index and $\mathbf{c}$ Simpson index of the urine microbiome from stone UR and tumor UR and controls. $\mathbf{d}$ The Chao1 and Ace indices and community richness were estimated among three groups. e A Venn diagram exhibiting the shared and unique OTUs among three groups. $\mathbf{f}$ PCoA of unweighted UniFrac distance is depicted for UR and healthy controls 
higher than that in controls (Fig. 1d, Chao1: controls vs tumor $\mathrm{UR}, \mathrm{P}=1.25 \times 10^{-3}$; controls vs tumor UR, $\mathrm{P}=6.68 \times 10^{-5}$; stone $\mathrm{UR}$ vs tumor $\mathrm{UR}, \mathrm{P}=0.15$; Ace: controls vs tumor UR, $\mathrm{P}=1.18 \times 10^{-3}$; controls vs tumor UR, $\mathrm{P}=7.41 \times 10^{-5}$; stone UR vs tumor $\mathrm{UR}, \mathrm{P}=0.19$ ). In addition, a Venn diagram (Fig. 1e) was used to characterize the overlapped OTUs among the three groups. A total of 212 OTUs were shared among the 3 groups, and there were 20,3 , and 2 OTUs were unique to the controls, stone UR and tumor UR groups, respectively. It is worth noting that there were as many as 42 OTUs shared between the stone UR and tumor UR groups.

To further investigate whether there were differences among the three groups in the urine microbiota spectrum, PCoA was performed based on the unweighted UniFrac distances of the $16 \mathrm{~S}$ rRNA sequence at the OTU level. There were differences in $\beta$-diversity among the three groups, as shown in Fig. If (PERMANOVA, pseudo-F statistic: $11.88, \mathrm{P}=1.00 \times 10^{-3}$ ). In addition, differences between each of the two groups were further evaluated based on unweighted and weighted UniFrac distances and Bray-Curtis dissimilarity (Additional file 3: Table S3). These results showed that both UR groups were significantly different from the control group, while the microbial compositions of two types of obstructive UR could not be separated.

\section{Altered urine microbial communities in stone UR and tumor UR}

To explore the specific microbial signature of stone UR and tumor UR, we evaluated the relative abundance of taxa in three groups. Urine microflora composition in each sample from three groups at phylum and genus levels are shown in the Additional file 4: Figure S1A, D. Average composition of bacterial community at the phylum and genus levels are presented in Additional file 4: Figure S1B, E, among which the genus level list the top 35 bacterial and all the remaining low-abundance microflora are combined into "Others". We found that Proteobacteria was the most abundant phylum in three groups, followed by Bacteroidetes (Additional file 4: Figure S1B). Compared with controls, stone UR and tumor UR individuals exhibited a significant increase in the phylum Bacteroidetes in urine (Additional file 4: Figure S1C). In accordance with the phylum level, the urine microbial compositions of the two types of obstructive UR patients were similar at the genus level, but both were different from that of the control group (Additional file 4: Figure S1E, F). It is noteworthy that there was a total of 44 bacteria with significant differences between any two of the three groups at the genus level $(\mathrm{q}<0.01$, Wilcoxon rank sum test, Fig. 2a). Twelve out of the 44 are displayed in Fig. 2b. Elizabethkingia, Proteus, Sphingomonas,
Pseudomonas, Acinetobacter, Sphingobacterium and Myroides were overrepresented in the stone UR and tumor UR groups. In contrast, Lactobacillus, Streptococcus, Gardnerella, Prevotella and Atopobium, which were decreased in stone UR and tumor UR patients, were enriched in controls.

To further confirm the specific bacteria associated with obstructive UR, LEfSe was used, which identified 14 discriminative features, and their relative abundances significantly varied between stone UR individuals and controls, which was completely consistent with the result in Fig. 2a, as evaluated by the Wilcoxon rank sum test (Additional file 5: Figure S2A). Curvibacter was a newly found bacterium enriched in the tumor UR group, while Escherichia was found enriched in control group (Additional file 5: Figure S2B).

Evaluation of the connections among these different genera was performed by a Spearman correlation test. Significant positive correlations were found in genera enriched obstructive UR, such as for Gluconacetobacter and Myroides $\left(\mathrm{R}=0.98, \mathrm{P}=1.24 \times 10^{-60}\right)$; Gluconacetobacter and Sphingobacterium $(\mathrm{R}=0.94$, $\left.\mathrm{P}=2.97 \times 10^{-40}\right) ; \quad$ Pseudomonas and Comamonas $\left(\mathrm{R}=0.85, \mathrm{P}=6.08 \times 10^{-24}\right)$; and so on. Likewise, positive correlations were also found in genera that were enriched in control subjects. More interestingly, the bacteria enriched in urine of the two obstructive UR patients were negatively correlated with those enriched in controls (Fig. 2c), such as Myroides and Lactobacillus ( $\mathrm{R}=-0.71$, $\left.\mathrm{P}=7.93 \times 10^{-14}\right)$; Sphingobacterium and Lactobacillus $\left(\mathrm{R}=-0.68, \mathrm{P}=3.45 \times 10^{-12}\right)$; Elizabethkingia and Atopobium $\left(\mathrm{R}=-0.46, \mathrm{P}=1.44 \times 10^{-5}\right)$; and so on. We further explored the association of the urine microbiome with clinical manifestations (Fig. 2d) and found that there were some significantly negative correlations between the estimated glomerular filtration rate (eGFR) level and the microorganisms that were enriched in obstructive UR patients, such as Pseudomonas, Methylobacterium, Elizabethkingia, and so on. On the contrary, positive correlations were observed between the uric acid (UA) level and the genera that were enriched in obstructive UR patients, such as Pseudomonas, Stenotrophomonas, Sphingomonas, and so on.

\section{Classification of disease status using bacterial genus-level biomarkers}

To explore the potential diagnostic value of the urine microbiome in stone UR and tumor UR, we constructed a random forest classifier to discriminate urinary retention samples from control samples. We only selected the microorganisms that were significantly enriched in stone UR and tumor UR to construct the classification models. Finally, 30 and 34 genera signatures were selected 


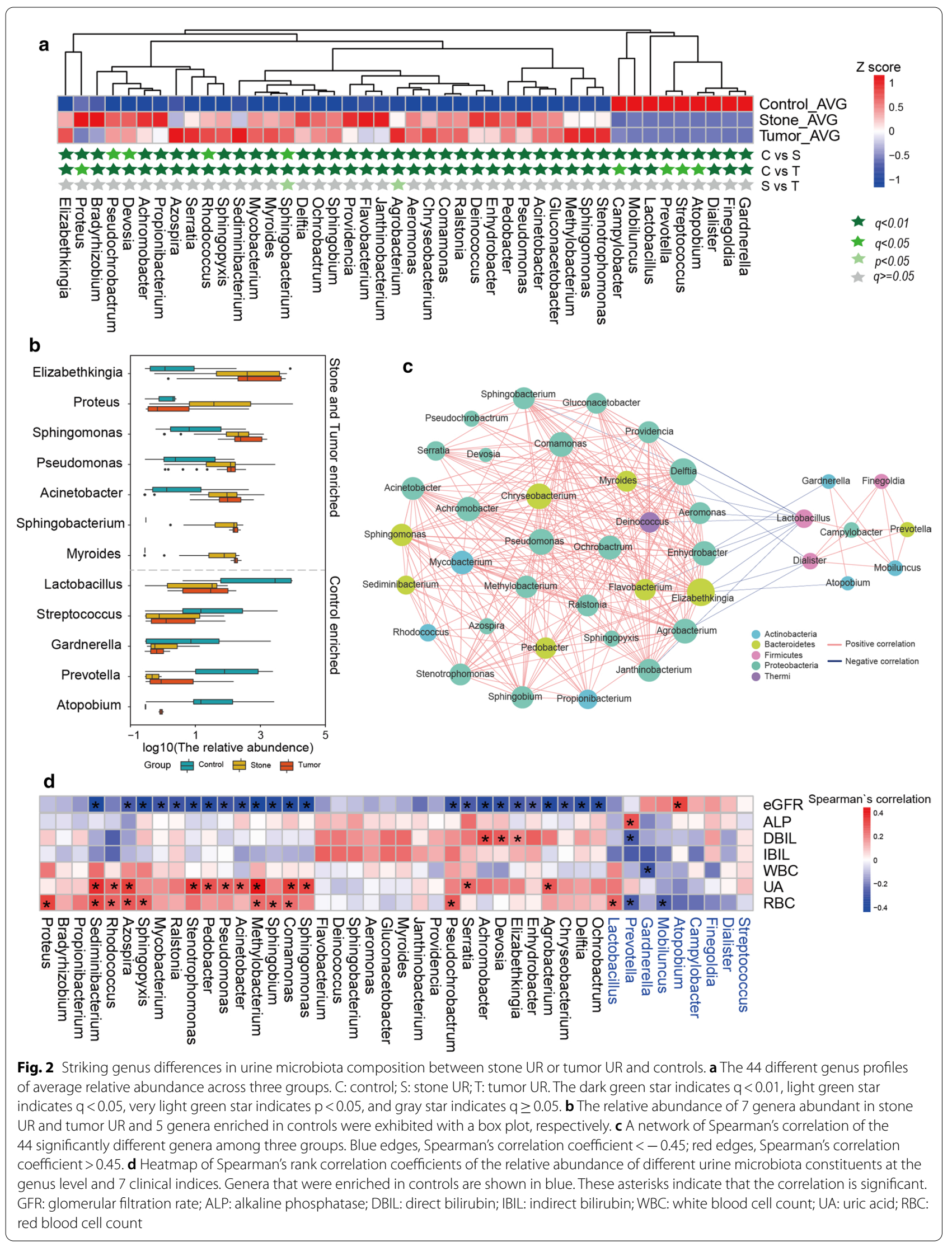


for further analysis for stone UR and tumor UR, respectively. The cross-validation error curve distribution was obtained from five trials of five-fold cross-validation. Both 8 biomarkers were selected as the optimal marker set to distinguish stone UR or tumor UR from the control group (Additional file 6: Figure S3A, B). The performance of these optimal marker models was assessed by 100 random ROC analyses, and the average AUC value achieved 92.29\% between the stone UR and control group and 97.96\% between the tumor UR and control group (Fig. 3a, d). The average MDA for the random 100 times of these optimal markers are shown in Fig. $3 \mathrm{~b}$ and e. These results showed that 7 out of 8 bacteria were identical, which were used to distinguish stone UR or tumor UR from the control group, including Mycobacterium, Agrobacterium, Ralstonia, Delftia, Acinetobacter, Methylobacterium and Sphingomonas. Here, we further validated our previous finding that stone UR and tumor UR have approximate urine microbial background although they result from different obstruction causes. The POD value was significantly increased in stone UR group versus control group, and a similar trend was also found in tumor UR group (Fig. 3c, f; $\mathrm{P}<2.2 \times 10^{-16}$ for both comparisons).

To select the optimal marker set of stone UR and tumor UR based on the urine microbiome, we used the genera with $\mathrm{P}<0.05$ which were measured by the Wilcoxon rank sum test. Only Sediminibacterium was selected from two different bacteria as the optimal marker after five

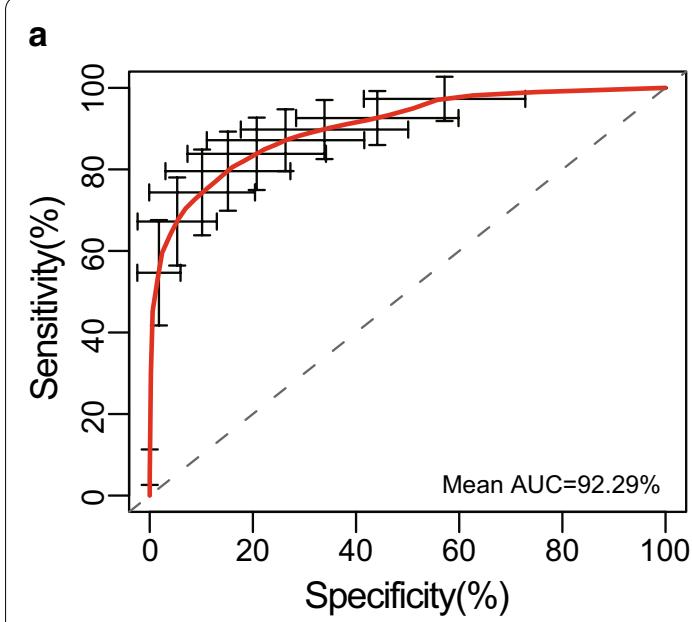

b
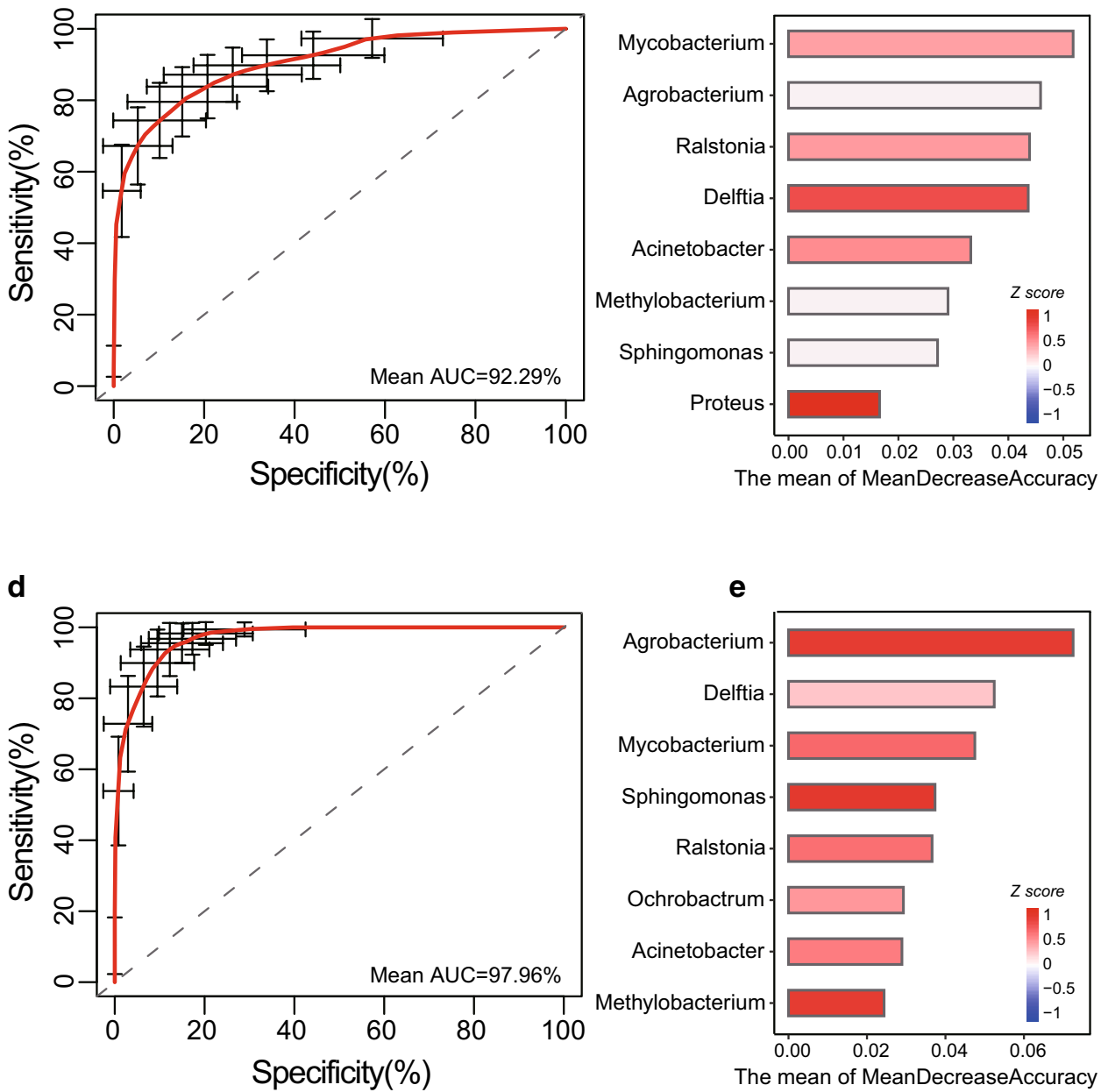

e

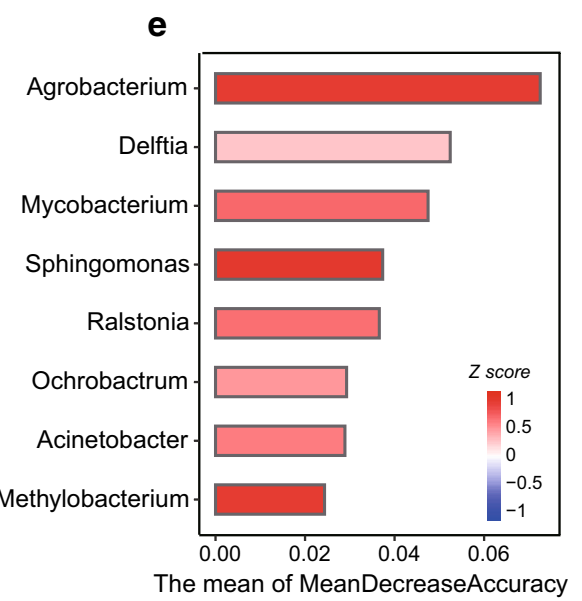

c

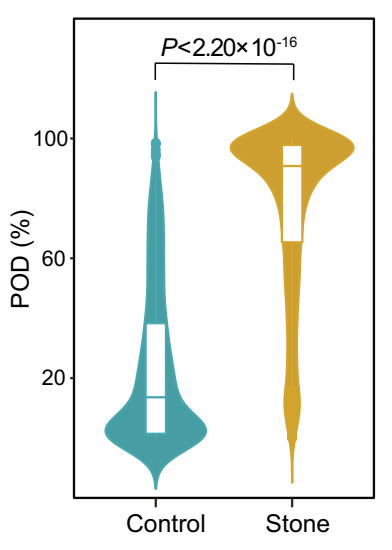

f

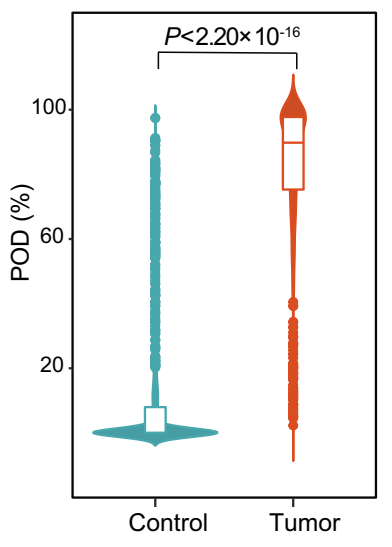

Fig. 3 Microbial genus-based biomarkers of UR classification by a random forest model. a The average AUC value was calculated 100 times between stone UR and controls. $\mathbf{b}$, e The optimal marker set was selected to distinguish stone UR or tumor UR from controls. The $\mathrm{x}$-axis of the histogram represents the MDA. The color of each bar is the z value normalized by "heatmap" R package for the relative abundance of each bacterium. c The box figure shows the POD value between the stone UR and controls. $\mathbf{d}$ The average AUC reached $97.96 \%$ by the same method as above. $\mathbf{f}$ The POD value of the tumor UR group and controls 
repeated five-fold cross-validation trials Additional file 6: Figure $\mathrm{S3C}$ ). The average AUC value was $58.44 \%$ after running 100 random ROC analyses (Additional file 6: Figure S3D). The MDA of Sediminibacterium is shown in Additional file 6: Figure S3E. The probability of tumor UR was significantly higher than stone UR in the tumor UR group (stone UR vs tumor UR, $\left.\mathrm{P}=1.00 \times 10^{-15}\right)$, which was consistent with these clinical data (Additional file 6: Figure S3F).

\section{Microbial functional altered in stone UR and tumor UR}

To explore whether the function of the urine microbiome of stone UR and tumor UR has changed, we used PICRUSt to predict the functional components of the $16 \mathrm{~S}$ rRNA gene sequencing data of all samples. The OTU profile of these three groups was aligned to level 3 of the KEGG database, and COG abundance was calculated (Additional file 7: Table S4, Additional file 8: Table S5). PCOA based on the KEGG pathways showed that the control group was strikingly separated from stone UR and tumor UR groups (Fig. 4a; PERMANOVA, pseudoF statistic: $13.05, \mathrm{P}=1.00 \times 10^{-3}$ ), which was consistent with the result based on COG categories (Additional file 9: Figure S4A; PERMANOVA, pseudo-F statistic: 11.97, $\mathrm{P}=1.00 \times 10^{-3}$ ). We found that both obstructive UR groups were significantly different from control group, while the functional structure between stone UR and tumor UR was similar (Fig. 4b). Twenty-five KEGG pathways were differentially enriched between each two of the three groups (adjusted p-value $<0.01$, Wilcoxon rank sum test). There were 14 pathways involved in membrane transport, signal transduction, genetic information processing, carbohydrate metabolism and nucleotide metabolism, such as that for purine and methane, which were significantly reduced in the stone UR group and tumor UR group. We observed 11 pathways that were increased in the stone UR and tumor UR groups, including amino acid metabolism and energy metabolism. Intriguingly, the abundance of pathways associated with membrane transport functions, such as $A B C$ transporters and the phosphotransferase system (PTS), was negatively correlated with these genera enriched in stone UR and tumor UR groups. In accordance with the KEGG function result, most of the energy metabolism, carbohydrate metabolism and amino acid metabolism pathways had changed in the COG annotation (Additional file 9: Figure S4B). There was a strong positive correlation between the significantly enriched functions in both obstructive UR groups and their enriched genera (Fig. 4c). For instance, Pseudomonas, Acinetobacter and Sphingomonas were significantly related to valine, leucine and isoleucine degradation and glycine, serine and threonine metabolism. These amino acids may be the product of bacteria as Corynebacterium glutamicum can produce amino acids on a large scale reported previously [34]. $\mathrm{ABC}$ transporters, the PTS, transporters and other ioncoupled transporters that were significantly enriched in controls were mainly negatively correlated with patients with UR microbial features. The same trend was found in the COG function analysis with these significantly different bacteria (Additional file 9: Figure S4C).

\section{Discussion}

This current study aims to analyze the microbial spectrum of two common types of obstructive urinary retention using 16S rRNA gene sequencing technology, a widely recognized technique that can depict the landscape of microorganisms. Our results showed that the microbial diversity of stone UR and tumor UR patients increased significantly compared with that of controls, while there was no obvious difference between these two types of obstructive UR. Changes in microbiome diversity of urinary system disorders have not been consistently. Previous studies have found increased microbial diversity in UUI [35] and chronic prostatitis patients [17] and decreased bacterial diversity in subjects with IC [16]. In addition, some urinary system diseases, such as overactive bladder [36], had no significant changes in microbial diversity. The increased community richness and diversity of the urine microbiome in stone UR and tumor UR groups is consistent with previous findings [37, 38] . Although the urine microbiota profile differs among individuals, which mainly due to genetic background, age, dietary habits and lifestyle, samples with same clinical phenotype was clustered in $\beta$-diversity analysis for their common urine microbial composition.

We observed a dramatic shift in the components of the urine microbiome in patients with two types of obstructive UR, however the urine microbiota feature of the stone UR and tumor UR patients were similar. A gram-negative genus, Pseudomonas, is the second most common infection in hospitalized patients. In addition, Pseudomonas is known to be associated with a wide range of urinary tract diseases [39-41]. In 2015, BarrBeare et al. not only detected Pseudomonas by sequencing from a urinary stone but also isolated this bacterium from stone culture [39]. Used 454 sequencing technology, $\mathrm{Xu}$ et al. detected that Pseudomonas was the dominant genus in some patients with urothelial carcinoma [42]. This finding agreed with previous studies that demonstrated that Pseudomonas spp. were enriched in stone UR and tumor UR patients. Acinetobacter is a complex genus, the virulence factors of Acinetobacter baumannii have been proofed to be involved in epithelial cell adhesion and invasion, biofilm formation [43]. A study profiling the urinary microbiome in man with calcium-based 


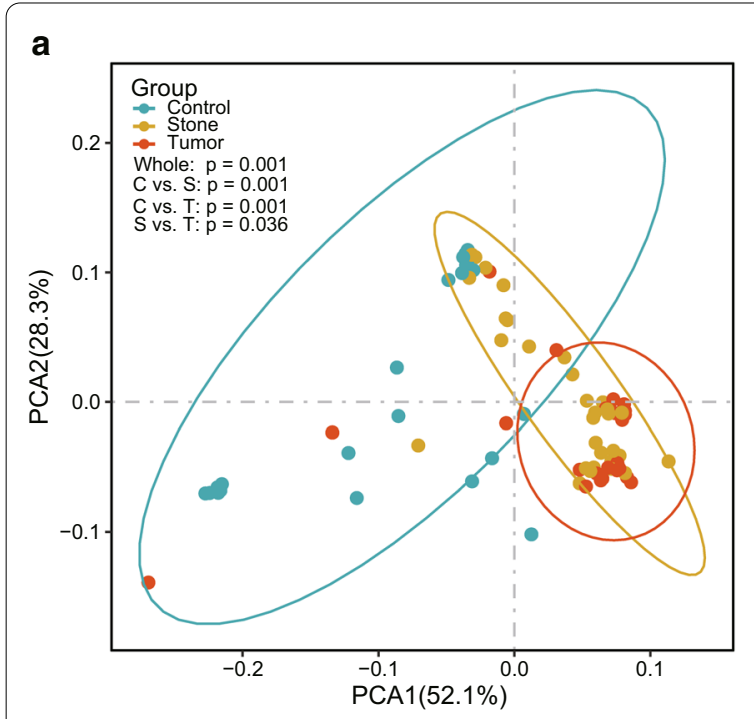

C
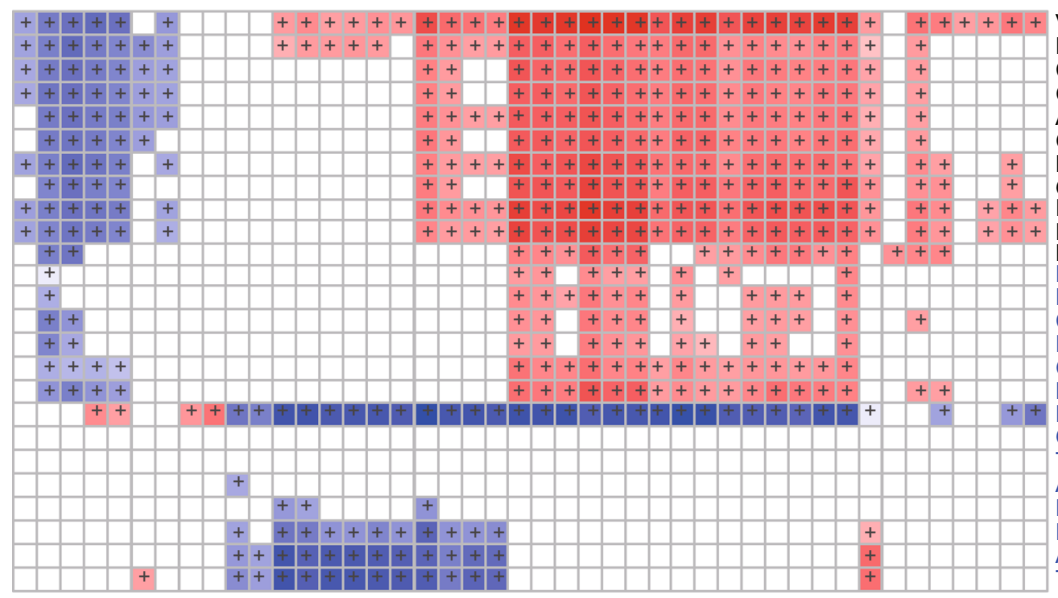

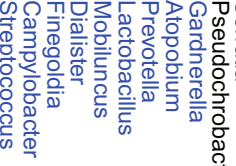
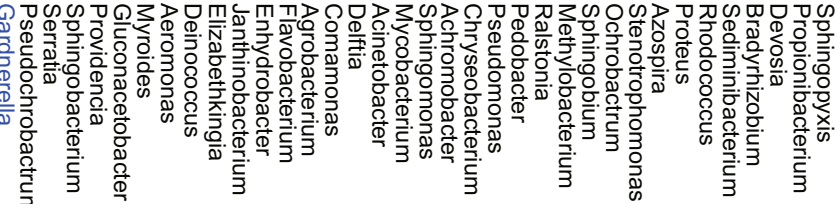

Fig. 4 Microbial function profiles of the UR patients and control individuals. a The relative abundance of level 3 KEGG database pathways in all 82 samples is shown by PCOA. $\mathbf{b}$ A total of 25 KEGG pathways were significantly different among three groups, based on the average relative abundance of the KEGG (level 3) pathway. $\mathbf{c}$ Heatmap of Spearman's correlation of the 44 significantly different genera and 25 significantly different pathways among three groups $(|r|>0.3)$. The genera or pathways that were enriched in the controls are shown in blue. These " + "indicate that the correlation is significant

kidney stone by Xie et al., which shown the most differentially represented taxa at genus level was Acinetobacter and enrichment in kidney stone patients [44]. Furthermore, Acinetobacter was an abundant genus in urothelial carcinoma patients and in male patients with bladder cancer in China [42, 45]. Sphingobacterium and Sphingomonas, which were also elevated in the tumor
UR group, are morbidity-inducing urine bacteria that cause bladder cancer, prostate cancer and BPH $[38,46]$. In addition, we found two unusual bacteria. The first is Myroides, which was the cause of an outbreak of UTI in a Tunisian hospital [47]. Almost all patients infected with Myroides spp. had a urinary stone or urinary neoplasms. Elizabethkingia was the second genus, and in 2017, a 
25-year-old woman was the first individual reported to have a UTI caused by Elizabethkingia and to have difficulty urinating [48]. On ground of our findings and previous reports, we assume that there is a strong association between obstructive UR and UTI, but it is not clear whether there is a causal relationship between them and what role microorganisms play. Furthermore, our results also indicated that a complex bacterial community dominated by Lactobacillus, Prevotella, Streptococcus and Gardnerella exists in the urinary tract of healthy people. These microorganisms have been speculated in previous studies to be mandatary to maintain a healthy status in the urinary system $[11,13,20,49,50]$. Lactobacillus plays an important role in the human body because it can protect the host from potential pathogens and maintaining urinary health [51]. Streptococcus has been found repeatedly in the urine of healthy men [52, 53]. A 2018 study showed that increased of Streptococcus in healthy bladder midstream urine versus the bladder cancer [37]. In addition, the microbial population profile of another urologic malignancy, prostate cancer, also found significant enrichment of Streptococcus in the non-tumor tissue [54]. Xu et al. detected the abundance of Streptococcus was near zero in most control patients but significantly elevated in urothelial carcinoma patients, however, which is the opposite of what was observed in our study [42]. Therefore, needed more research to elucidate the association between Streptococcus and the cancer or health status. It is worth mentioning that these beneficial microbial enriched in control group were negatively correlated with the pathogenic bacteria in the stone UR and tumor UR groups but positively correlated internally. Of interest, products of some bacteria might inhibit the activity of others. For example, lactic acid produced by Lactobacillus showed good inhibition activity against E. coli [55].

Stone UR and tumor UR microbial anomalies were characterized by altered relative abundance in 44 genera. The combination of the optimal marker taxa that distinguished stone UR or tumor UR patients from healthy controls had high accuracies of $92.29 \%$ and $97.96 \%$, respectively. It suggests that these differentially present microbial communities may be a potentially effective tool for predicting stone UR or tumor UR, and further combination with clinical information may enhance the identification capability. Further detailed studies are needed to obtain better classifiers to distinguish stone UR and tumor UR in the future.

Along with the altered composition of the urine microbiome, their functional change was also predicted by PICRUSt. Biosynthesis and metabolism of some carbohydrates and nucleotides were depleted in obstructive UR, such as amino and nucleotide sugar metabolism, fructose and mannose metabolism and glycolysis/gluconeogenesis, which are essential for the host. Pathway analysis also showed a decline in the capacity for membrane transport and signal transduction, indicating impaired membrane permeability or ureteral obstruction. Ureteral obstruction leads to a gradual decrease in renal excretion with decreased eGFR levels [56]. In our cohort, the majority of pathogenic genera in stone UR and tumor UR were negatively correlated with eGFR levels, suggesting a potential link between these microbes and disease severity. Similarly, many pathogens of UR were significantly positively correlated with UA content. Clinical knowledge suggests that the elevated UA content is a result of host purine metabolism disorder. Purine metabolism was reduced in patients with obstructive UR, as expected, hinting at a potential role of urine microbes in causing nucleotide metabolism dysfunction. Although the purine metabolism ability was weakened in two UR groups, UA content could not be excreted due to obvious impairment of membrane transport, which showed that UA levels of these two obstructive UR patients were higher than those of control individuals (Table 1).

There are still several limitations in our study to be addressed. First, it is difficult to determine the causal relationship between the microflora and obstructive UR. Therefore, prospective follow-up studies with larger sample sizes and experimental studies are needed to detect the role of the microbiome in obstructive UR progression and development. Second, our pathway and function characterizations were inferred by PICRUSt based on the $16 \mathrm{~S}$ rRNA sequence. In the future, the combination of metagenomics and metaproteomics may reveal more accurate microbial community composition and function.

\section{Conclusions}

In summary, we characterized the microbiome profiles of two obstructive UR, stone UR and tumor UR, in comparison with control subjects. Our study suggested that the urine microbiome may be associated with obstructive UR, while the cause-effect relationship remains to be elucidated. Some bacteria can be used to discriminate obstructive UR patients from healthy individuals with high accuracy. A better understanding of the role of the urinary microflora in obstructive UR would help urologists make more sensible choices in clinical identification and formulate personalized microbial intervention measures. 


\section{Supplementary Information}

The online version contains supplementary material available at https://doi. org/10.1186/s12941-020-00408-5.

Additional file 1: Table S1. Data production of 84 samples in three groups.

Additional file 2: Table S2. The relative abundance of the OTU profile and taxonomy.

Additional file 3: Table S3. PERMANOVA tests of the urine microbiota at different dissimilarity distances.

Additional file 4: Figure S1. Phylogenetic profiles of urine microbes among stone UR and tumor UR and controls. (A, D) The microflora composition abundance of each sample at the phylum level (A) and genus level (D). (B, E) Composition of the urine microbiota at the phylum level (B) and genus level (E) among three groups. (C) The 5 different phylum profiles of average relative abundance across three groups tested by the Wilcoxon rank sum test. The dark green star indicates $\mathrm{q}<0.01$, light green star indicates $q<0.05$, very light green star indicates $p<0.05$, and gray star indicates $q \geq 0.05$. (F) PCoA analysis based on genus level of for two types UR and healthy controls.

Additional file 5: Figure S2. LEfSe analyses of the urine microbiomes of stone UR and tumor UR patients compared to those of controls. (A) The LDA bar graphs show differential abundance between stone UR and controls. (B) The LDA bar graphs show differential abundance between tumor UR and controls.

Additional file 6: Figure S3. A random forest model was established to distinguish stone UR from tumor UR based on microbial genus. (A, B, C) 8,8 and 1 genus were selected as the optimal marker set for the three 'randomForest' model respectively (A: controls vs stone UR; $\mathrm{B}$ : controls vs tumor UR; C: stone UR vs tumor UR). (D) The average AUC value after 100 ROC measurements to distinguish two types of UR. (E) The MDA of this marker between stone UR and tumor UR. (F) The probability of tumor UR value between two types of obstructive UR.

Additional file 7: Table S4. The relative abundance of level 3 KEGG pathway profiles predicted by PICRUSt.

Additional file 8: Table S5. The relative abundance of level 2 COG functions predicted by PICRUSt.

Additional file 9: Figure S4. Microbial function profiles of these UR patients and the control subjects. (A) PCOA analysis for the relative abundance of level 2 COG categories of all participants. (B) A total of 18 COG functions were significantly different among three groups. (C) Heatmap of Spearman's correlation of the 44 significantly different genera and 18 significantly different pathways among three groups. The genera or pathways that were enriched in controls are shown in blue.

\section{Abbreviations}

UR: Urinary retention; BPH: Benign prostatic hyperplasia; UI: Urinary incontinence; UTI: Urinary tract infection; UUI: Urgency urinary incontinence; IC: Interstitial cystitis; CP/CPPS: Chronic prostatitis/chronic pelvic pain syndrome; USD: Urinary stone disease; EQUC: Expanded quantitative urine culture; OTU: Operational taxonomic unit; QIIME: Quantitative Insights Into Microbial Ecology; LefSe: Linear discriminant analysis for effect size; PICRUSt: Phylogenetic Investigation of Communities by Reconstruction of Unobserved States; PCoA: Principal coordinate analysis; PERMANOVA: Permutational multivariate analysis of variance; ROC: Receiver operating characteristic curve; AUC: Area under the curve; MDA: Mean decrease accuracy; POD: Probability of disease; KEGG: Kyoto Encyclopedia of Genes and Genomes; COG: Orthologous Groups of proteins; PTS: Phosphotransferase system.

\section{Acknowledgements}

Authors would like to thanks the team of Jinyu Wu from the Institute of Genomic Medicine, Wenzhou Medical University for supporting the work.

\section{Authors' contributions}

All authors were involved in drafting the article or revising it critically for important intellectual content, and all authors approved the final version to be published. XW and SX conceived the project and revised the manuscript. $S J$ did the data processing and wrote the manuscript. SL and $Y Z$ organized recruitment, sample collection and medication data. XC, FL and CZ participate in the data analysis and interpretation. All authors read and approved the final manuscript.

\section{Funding}

This study was supported by the National Natural Science Foundation of China (81700062), the Natural Science Foundation of Zhejiang Province grants (LQ16H010003 and LY18H030011), and Science and Technology Project of Zhejiang Provincial Health Commission (2019RC050).

\section{Availability of data and materials}

The datasets used and/or analyzed in this study are available from the corresponding author on reasonable request.

\section{Ethics approval and consent to participate}

All participants gave written informed consent and this study was approved by the Ethical Committee of the First Affiliated Hospital of Wenzhou Medical University (approval \# 19071).

\section{Consent for publication}

No applicable.

\section{Competing interests}

The authors have no conflicts of interest to declare.

\section{Author details}

${ }^{1}$ Institute of Genomic Medicine, Wenzhou Medical University, Wenzhou, China. ${ }^{2}$ Department of Rheumatology, The First Affiliated Hospital of Wenzhou Medical University, Wenzhou, China. ${ }^{3}$ Department of Urology, The First Affiliated Hospital of Wenzhou Medical University, Wenzhou, China. ${ }^{4}$ Department of Ultrasonography, The First Affiliated Hospital of Wenzhou Medical University, Wenzhou, China.

Received: 11 September 2020 Accepted: 8 December 2020

Published online: 06 January 2021

References

1. De La Cruz MS, Buchanan EM. Uterine fibroids: diagnosis and treatment. Am Fam Physician. 2017;95(2):100-7.

2. Choong S, Emberton M. Acute urinary retention. BJU Int. 2000;85(2):186-201.

3. Murray K, Massey A, Feneley RC. Acute urinary retention-a urodynamic assessment. Br J Urol. 1984;56(5):468-73.

4. Patel R, Nitti V. Bladder outlet obstruction in women: prevalence, recognition, and management. Curr Urol Rep. 2001;2(5):379-87.

5. Dawam D, Rafindadi AH, Kalayi GD. Benign prostatic hyperplasia and prostate carcinoma in native Africans. BJU Int. 2000;85(9):1074-7.

6. Ferentinos P, Margaritis D, Douzenis A. Escitalopram-associated acute urinary retention in elderly men with known or latent Benign Prostatic Hyperplasia: a case series. Clin Neuropharmacol. 2016;39(6):327-8.

7. Patel JA, Kaufman AS, Howard RS, Rodriguez CJ, Jessie EM. Risk factors for urinary retention after laparoscopic inguinal hernia repairs. Surg Endosc. 2015;29(11):3140-5

8. Lombardi G, Musco S, Celso M, Del Corso F, Del Popolo G. Sacral neuromodulation for neurogenic non-obstructive urinary retention in incomplete spinal cord patients: a ten-year follow-up single-centre experience. Spinal Cord. 2014;52(3):241-5.

9. Selius BA, Subedi R. Urinary retention in adults: diagnosis and initial management. Am Fam Physician. 2008;77(5):643-50.

10. Lewis DA, Brown R, Williams J, White P, Jacobson SK, Marchesi JR, Drake MJ. The human urinary microbiome; bacterial DNA in voided urine of asymptomatic adults. Front Cell Infect Microbiol. 2013;3:41. 
11. Wolfe AJ, Toh E, Shibata N, Rong R, Kenton K, Fitzgerald M, Mueller ER, Schreckenberger P, Dong Q, Nelson DE, et al. Evidence of uncultivated bacteria in the adult female bladder. J Clin Microbiol. 2012;50(4):1376-83.

12. Nelson DE, Dong Q, Van der Pol B, Toh E, Fan B, Katz BP, Mi D, Rong $R$, Weinstock GM, Sodergren E, et al. Bacterial communities of the coronal sulcus and distal urethra of adolescent males. PLoS ONE. 2012;7(5):e36298.

13. Siddiqui $H$, Nederbragt AJ, Lagesen $K$, Jeansson $S L$, Jakobsen KS. Assessing diversity of the female urine microbiota by high throughput sequencing of 16S rDNA amplicons. BMC Microbiol. 2011;11:244.

14. Foxman B. The epidemiology of urinary tract infection. Nat Rev Urol. 2010;7(12):653-60

15. Pearce MM, Hilt EE, Rosenfeld AB, Zilliox MJ, Thomas-White K, Fok C, Kliethermes S, Schreckenberger PC, Brubaker L, Gai X, et al. The female urinary microbiome: a comparison of women with and without urgency urinary incontinence. mBio. 2014;5(4):e01283.

16. Siddiqui $H$, Lagesen $K$, Nederbragt AJ, Jeansson SL, Jakobsen KS. Alterations of microbiota in urine from women with interstitial cystitis. BMC Microbiol. 2012;12:205

17. Shoskes DA, Altemus J, Polackwich AS, Tucky B, Wang H, Eng C. The urinary microbiome differs significantly between patients with chronic prostatitis/chronic pelvic pain syndrome and controls as well as between patients with different clinical phenotypes. Urology. 2016:92:26-32.

18. Dornbier RA, Bajic P, Van Kuiken M, Jardaneh A, Lin H, Gao X, Knudsen B, Dong Q, Wolfe AJ, Schwaderer AL. The microbiome of calcium-based urinary stones. Urolithiasis. 2020;48(3):191-9.

19. Zampini A, Nguyen AH, Rose E, Monga M, Miller AW. Defining dysbiosis in patients with urolithiasis. Sci Rep. 2019;9(1):5425.

20. Fouts DE, Pieper R, Szpakowski S, Pohl H, Knoblach S, Suh MJ, Huang ST, Ljungberg I, Sprague BM, Lucas SK, et al. Integrated next-generation sequencing of $16 \mathrm{~S}$ rDNA and metaproteomics differentiate the healthy urine microbiome from asymptomatic bacteriuria in neuropathic bladder associated with spinal cord injury. J Transl Med. 2012;10:174

21. Wang T, Liu Q, Li X, Wang X, Li J, Zhu X, Sun ZS, Wu J. RRBS-analyser: a comprehensive web server for reduced representation bisulfite sequencing data analysis. Hum Mutat. 2013;34(12):1606-10.

22. Liu Q, Chen C, Shen E, Zhao F, Sun Z, Wu J. Detection, annotation and visualization of alternative splicing from RNA-Seq data with SplicingViewer. Genomics. 2012;99(3):178-82

23. Masella AP, Bartram AK, Truszkowski JM, Brown DG, Neufeld JD. PANDAseq: paired-end assembler for illumina sequences. BMC Bioinform. 2012;13:31

24. Caporaso JG, Kuczynski J, Stombaugh J, Bittinger K, Bushman FD, Costello EK, Fierer N, Pena AG, Goodrich JK, Gordon Jl, et al. QIIME allows analysis of high-throughput community sequencing data. Nat Methods. 2010;7(5):335-6.

25. DeSantis TZ, Hugenholtz P, Larsen N, Rojas M, Brodie EL, Keller K, Huber T, Dalevi D, Hu P, Andersen GL. Greengenes, a chimera-checked 16S rRNA gene database and workbench compatible with ARB. Appl Environ Microbiol. 2006;72(7):5069-72

26. Edgar RC. UPARSE: highly accurate OTU sequences from microbial amplicon reads. Nat Methods. 2013;10(10):996-8.

27. Lozupone C, Knight R. UniFrac: a new phylogenetic method for comparing microbial communities. Appl Environ Microbiol. 2005;71(12):8228-35

28. Segata N, Izard J, Waldron L, Gevers D, Miropolsky L, Garrett WS, Huttenhower C. Metagenomic biomarker discovery and explanation. Genome Biol. 2011;12(6):R60

29. Szafranski SP, Wos-Oxley ML, Vilchez-Vargas R, Jauregui R, Plumeier I, Klawonn F, Tomasch J, Meisinger C, Kuhnisch J, Sztajer H, et al. Highresolution taxonomic profiling of the subgingival microbiome for biomarker discovery and periodontitis diagnosis. Appl Environ Microbiol. 2015:81(3):1047-58.

30. Langille MG, Zaneveld J, Caporaso JG, McDonald D, Knights D, Reyes JA, Clemente JC, Burkepile DE, Vega Thurber RL, Knight R, et al. Predictive functional profiling of microbial communities using 165 rRNA marker gene sequences. Nat Biotechnol. 2013;31(9):814-21.

31. Qin N, Yang F, Li A, Prifti E, Chen Y, Shao L, Guo J, Le Chatelier E, Yao J, Wu $\mathrm{L}$, et al. Alterations of the human gut microbiome in liver cirrhosis. Nature. 2014:513(7516):59-64.
32. Li J, Zhao F, Wang Y, Chen J, Tao J, Tian G, Wu S, Liu W, Cui Q, Geng B, et al. Gut microbiota dysbiosis contributes to the development of hypertension. Microbiome. 2017;5(1):14.

33. Ren Z, Li A, Jiang J, Zhou L, Yu Z, Lu H, Xie H, Chen X, Shao L, Zhang $R$, et al. Gut microbiome analysis as a tool towards targeted non-invasive biomarkers for early hepatocellular carcinoma. Gut. 2019:68(6):1014-23.

34. Liu G, Wu J, Yang H, Bao Q. Codon usage patterns in Corynebacterium glutamicum: mutational bias, natural selection and amino acid conservation. Comp Funct Genomics. 2010;2010:343569.

35. Thomas-White KJ, Hilt EE, Fok C, Pearce MM, Mueller ER, Kliethermes S, Jacobs K, Zilliox MJ, Brincat C, Price TK, et al. Incontinence medication response relates to the female urinary microbiota. Int Urogynecol J. 2016:27(5):723-33.

36. Curtiss N, Balachandran A, Krska L, Peppiatt-Wildman C, Wildman S, Duckett J. A case controlled study examining the bladder microbiome in women with Overactive Bladder (OAB) and healthy controls. Eur J Obstet Gynecol Reprod Biol. 2017;214:31-5.

37. Bucevic Popovic V, Situm M, Chow CT, Chan LS, Roje B, Terzic J. The urinary microbiome associated with bladder cancer. Sci Rep. 2018;8(1):12157.

38. Wu P, Zhang G, Zhao J, Chen J, Chen Y, Huang W, Zhong J, Zeng J. Profiling the urinary microbiota in male patients with bladder cancer in China. Front Cell Infect Microbiol. 2018;8:167.

39. Barr-Beare E, Saxena V, Hilt EE, Thomas-White K, Schober M, Li B, Becknell B, Hains DS, Wolfe AJ, Schwaderer AL. The interaction between Enterobacteriaceae and calcium oxalate deposits. PLoS ONE. 2015;10(10):e0139575.

40. Willner D, Low S, Steen JA, George N, Nimmo GR, Schembri MA Hugenholtz $P$. Single clinical isolates from acute uncomplicated urinary tract infections are representative of dominant in situ populations. MBio. 2014;5(2):e01064.

41. Chen D, Zhang Y, Huang J, Liang X, Zeng T, Lan C, Duan X, Zhao Z Zeng G, Tiselius HG, et al. The analysis of microbial spectrum and antibiotic resistance of uropathogens isolated from patients with urinary stones. Int J Clin Pract. 2018;72(6):e13205.

42. Xu W, Yang L, Lee P, Huang WC, Nossa C, Ma Y, Deng FM, Zhou M, Melamed J, Pei Z. Mini-review: perspective of the microbiome in the pathogenesis of urothelial carcinoma. Am J Clin Exp Urol. 2014;2(1):57-61.

43. McConnell MJ, Actis L, Pachon J. Acinetobacter baumannii: human infections, factors contributing to pathogenesis and animal models. FEMS Microbiol Rev. 2013:37(2):130-55.

44. Xie J, Huang JS, Huang XJ, Peng JM, Yu Z, Yuan YQ, Xiao KF, Guo JN. Profiling the urinary microbiome in men with calcium-based kidney stones. BMC Microbiol. 2020;20(1):41.

45. Wu P, Zhang G, Zhao J, Chen J, Chen Y, Huang W, Zhong J, Zeng J. Corrigendum: profiling the urinary microbiota in male patients with bladder cancer in China. Front Cell Infect Microbiol. 2018;8:429.

46. Yu H, Meng H, Zhou F, Ni X, Shen S, Das UN. Urinary microbiota in patients with prostate cancer and benign prostatic hyperplasia. Arch Med Sci. 2015;11(2):385-94.

47. Ktari S, Mnif B, Koubaa M, Mahjoubi F, Ben Jemaa M, Mhiri MN, Hammami A. Nosocomial outbreak of Myroides odoratimimus urinary tract infection in a Tunisian hospital. J Hosp Infect. 2012;80(1):77-81.

48. Gupta P, Zaman K, Mohan B, Taneja N. Elizabethkingia miricola: a rare non-fermenter causing urinary tract infection. World J Clin Cases. 2017;5(5):187-90.

49. Karstens L, Asquith M, Davin S, Stauffer P, Fair D, Gregory WT, Rosenbaum JT, McWeeney SK, Nardos R. Does the urinary microbiome play a role in urgency urinary incontinence and its severity? Front Cell Infect Microbiol. 2016;6:78.

50. Hilt EE, McKinley K, Pearce MM, Rosenfeld AB, Zilliox MJ, Mueller ER, Brubaker L, Gai X, Wolfe AJ, Schreckenberger PC. Urine is not sterile: use of enhanced urine culture techniques to detect resident bacterial flora in the adult female bladder. J Clin Microbiol. 2014;52(3):871-6.

51. Martin R, Miquel S, Ulmer J, Kechaou N, Langella P, Bermudez-Humaran LG. Role of commensal and probiotic bacteria in human health: a focus on inflammatory bowel disease. Microb Cell Fact. 2013;12:71.

52. Aragon IM, Herrera-Imbroda B, Queipo-Ortuno MI, Castillo E, Del Moral JS, Gomez-Millan J, Yucel G, Lara MF. The urinary tract microbiome in health and disease. Eur Urol Focus. 2018:4(1):128-38. 
53. Dong Q, Nelson DE, Toh E, Diao L, Gao X, Fortenberry JD, Van der Pol B. The microbial communities in male first catch urine are highly similar to those in paired urethral swab specimens. PLoS ONE. 2011;6(5):e19709.

54. Cavarretta I, Ferrarese R, Cazzaniga W, Saita D, Luciano R, Ceresola ER, Locatelli I, Visconti L, Lavorgna G, Briganti A, et al. The microbiome of the prostate tumor microenvironment. Eur Urol. 2017;72(4):625-31.

55. Abedi D, Feizizadeh S, Akbari V, Jafarian-Dehkordi A. In vitro antibacterial and anti-adherence effects of Lactobacillus delbrueckii subsp. bulgaricus on Escherichia coli. Res Pharm Sci. 2013;8(4):260-8.
56. Jones DA, Atherton JC, O'Reilly PH, Barnard RJ, George NJ. Assessment of the nephron segments involved in post-obstructive diuresis in man, using lithium clearance. Br J Urol. 1989;64(6):559-63.

\section{Publisher's Note}

Springer Nature remains neutral with regard to jurisdictional claims in published maps and institutional affiliations.
Ready to submit your research? Choose BMC and benefit from:

- fast, convenient online submission

- thorough peer review by experienced researchers in your field

- rapid publication on acceptance

- support for research data, including large and complex data types

- gold Open Access which fosters wider collaboration and increased citations

- maximum visibility for your research: over 100M website views per year

At BMC, research is always in progress.

Learn more biomedcentral.com/submissions 